\title{
Modality specific word comprehension deficits in deep dyslexia
}

\author{
TIM SHALLICE AND ANTHONY K COUGHLAN
}

\begin{abstract}
From the MRC Applied Psychology Unit, Cambridge, and Department of Psychology, The National Hospital for Nervous Diseases, London, and the Wolfson Medical Rehabilitation Centre, London
\end{abstract}

SUMMARY A deep dyslexic patient was tested on a series of experiments designed to assess her comprehension of abstract words. On tests where a precise semantic representation of words was required, performance was much poorer with visual than with auditory presentation. However, on some but not all categorisation tests performance with both modalities was good. It is argued that deep dyslexia can result from a modality-specific deficit in attaining the meanings of words together with a disorder of the phonological reading route.

In recent years the classical analysis of the dyslexias in terms of the presence or absence of an associated dysgraphia has been increasingly replaced by an approach based on the analysis of the information-processing stages at which the deficits in reading occur. The investigation of one syndrome deep (or phonemic) dyslexia, has probably contributed most to this change-both because of its intuitively surprising characteristics and because it lends itself to analysis in information-processing terms.

There are three cardinal features of the syndrome-an inability to read by means of phonological transformation, that is sounding out (so that, for instance, nonsense syllables cannot be read), a very large effect of the part of speech and the semantic characteristics of a word on the chance of it being read (so that concrete words are read much more easily than abstract ones and nouns and adjectives more easily than verbs and function words), and a particular pattern of errors with words semantically, visually and derivationally related to the stimulus word all being produced as errors. The syndrome was first described by Marshall and Newcombe ${ }^{1}$ in patient GR. A number of other patients with closely similar characteristics have been described since 1975 by various authors (for review see Coltheart, ${ }^{2}$ and Shallice and Warrington ${ }^{3}$ ).

Interest in the syndrome has led to it being

Address for reprint requests: Tim Shallice, MRC Applied Psychology Unit, 15 Chaucer Road, Cambridge CB2 2EF, Cambridge.

Accepted 20 May 1980 used as a basis for speculations on the operation of the "direct" reading route, ${ }^{4}$ and on the functions of the right hemisphere-it has been held to reflect right hemisphere reading. ${ }^{5-7}$ Yet it is still not understood which informationprocessing systems need to be damaged for the syndrome to occur. Whilst impairment in the operation of the phonological reading route is a striking feature of the syndrome it cannot constitute a full explanation as specific deficits of this route can occur without yielding the full range of deficits found in deep dyslexia. ${ }^{3} 8$ One widely favoured candidate for another difficulty that is involved is a deficit in encoding the semantic representation of the word for naming (for example Marshall and Newcombe, ${ }^{1}$ Patterson $^{9}$ ). (This will be called the "naming hypothesis.") An alternative conception favoured by Shallice and Warrington ${ }^{10}$ is that there is a deficit in attaining the semantic representation of certain types of words given visual presentation in these patients. (This will be called the "semantic attainment" hypothesis.)

These two candidate hypotheses make different predictions about the written word comprehension performance of deep dyslexics for words they cannot read. On the naming hypothesis, the impairment would occur after comprehension and so there should be no comprehension deficit for written words. In contrast, on the semantic attainment hypothesis, there should be a comprehension deficit for written words, but not for spoken words. In the present paper we assess the comprehension abilities of a deep dyslexic patient. In the final section we investigate her 
ability to comprehend words by a number of different methods.

\section{Case history}

PS, a 23 year old right-handed teacher, was involved in a road traffic accident in September, 1971, in which she sustained a severe head injury with a compound depressed left fronto-temporo-parietal fracture. She was admitted to Plymouth General Hospital under the care of Mr HH Gossman and a debridement was performed. Severe contusion of the underlying brain was noted and a $2 \times 3 \mathrm{~cm}$ area of necrotic tissue was resected, most of this tissue coming from the posterior aspect of the inferior frontal gyrus (Broca's area). She regained consciousness slowly and it was 10 days before she could respond to simple verbal commands. The main clinical deficits arising from the accident were a very severe Broca's aphasia, a complete right-hemianopia, and a right-hemianaesthesia which have persisted, and a right hemiparesis which now principally involves the arm. After eight months she developed epileptic attacks and these have persisted, the attacks occurring once or twice a month. In September, 1974 she was briefly readmitted to hospital for a cosmetic acrylic cranioplasty of the left temporal area and it was then found that considerable atrophy of the temporal lobe had taken place.

PS was admitted to The Wolfson Medical Rehabilitation Centre for intensive rehabilitation on two occasions, the first in November, 1975 for 10 weeks and the second in April, 1977 for three weeks. During these admissions PS was referred for routine psychological assessment. She was assessed on the WAIS three times, in December 1975, January 1976 and May 1977 and obtained verbal IQs of 60,63 and 66 respectively and performance IQs of 78, 91 and 86 respectively. She also attempted Raven's Progressive Matrices and obtained a raw score of 32 (equivalent to IQ 85).

An independent assessment on the Boston Diagnostic Aphasia examination by Mrs Elizabeth Bridgman revealed the typical profile of a Broca's aphasic with the exception that articulatory agility was much better than that normally found amonzst Broca's aphasics. (Rating scale scores were as follows: Melodic line 4, Phrase Length 3, Articutory Agility 6, Grammatical Form 4, Paraphasia in running speech 7 , Word Finding 6.5, Auditory Comprehension $Z=+0 \cdot 5$ ). Word-retrieval skills were relatively well preserved: on the Coughlan and Warrington $^{11}$ test of Object Naming she scored $10 / 15$ (also 13/15) and $9 / 15$ on the test of naming objects from their verbal description. On the Oldfield pictures test she scored 17/26 (also 19/26). As noted above, her articulatory abilities were good as she could usually repeat familiar polysyllabic words without difficulty. Her digit span was always four digits forwards and two backwards. On the Lesser ${ }^{12}$ Syntax test she showed mild to moderate impairment $(61 / 80)$, making errors on a variety of syntactic features. On a modified version of the De Renzi and Vignolo13 token test (Coughlan and Warrington ${ }^{11}$ ) she scored 0/15. Even allowing for this her recall of details from a short story seemed poor. She did, though, score at an average level $(45 / 50)$ on a forcedchoice word-recognition memory test ${ }^{14}$ which employed visual presentation. On a dichotic listening test, she correctly reported $85 \%$ of digits (in strings of three) on the left ear, but only $12 \%$ of those on the right.

Visual functions appeared to be intact; she had no difficulty in recognising objects, scoring within normal limits on the Warrington and Taylor unusual views test.15 She was, however, impaired on Warrington and Sanders' forced-choice memory for faces test, ${ }^{14}$ scoring only $37 / 50$.

Among her aphasic difficulties, a reading defect has been investigated in greatest detail. Oral reading and spelling (both oral and written) were consistently poor, scores ranging between 25 and 28 out of 100 on the Schonell Graded Word Reading Test and scores on the Schonell Graded Word Spelling Test being $12 / 100$ and $14 / 100$ on the two occasions of testing. Apart from some hesitancy and occasional errors which she spontaneously corrected, letters and numbers were read correctly.

The more detailed account of her reading difficulties that follows is based on results obtained during her two admissions to The Wolfson Centre together with two further periods of testing in August 1977 and June 1978. During the entire period of December 1975 to June 1978 her language abilities appeared to be static, except for a minor improvement in her ability to use phonological recoding. This did not lead to any substantive improvement in reading as her 1978 Schonell score of 25/100 indicates.

\section{Studies of reading}

1 Basic reading performance PS was asked to read a set of 375 words (corpus I) comprising 335 words from the Brown and Ure ${ }^{16}$ list, 27 function words and 13 number names. The list also contained 15 pronounceable non-words of five or six letters each differing by one letter from an English word. All words were presented individually on index cards in a random order.

On this test she read 146 of the words correctly, made 175 substantive errors and 48 omissions. She produced paraphasic responses to six words. The pattern of words she read correctly on the Brown and Ure list analysed by concreteness (nouns only) is shown in table 1 . For comparison the results of KF (from Shallice and Warrington ${ }^{10}$ ), a previously studied deep dyslexic, on a larger set of Brown and Ure words are also given. Her performance is very similar to his. The concreteness effect is characteristic of deep dyslexia. Of unambiguous adjectives she read $14 / 33(42 \%)$ and of unambiguous verbs $5 / 23(22 \%)$. (The values for $\mathrm{KF}$ for analogous words were $32 \%$ and $7 \%$ respectively.) However, she read 11/27 $(40 \%)$ function words, a higher percentage than 
Table 1 The percentage of words normally used as nouns read correctly by PS from the set of 335 Brown and Ure words for three different concreteness ranges and two frequency ranges. The results of $K F$ using a set of words drawn from the same pool are also shown (from Shallice and Warrington. 10 In brackets is given the number of words from which the percentage is derived. VHC stands for Very High, MHC for Medium High and LC for Low Concreteness respectively

\begin{tabular}{|c|c|c|c|c|}
\hline & \multirow{2}{*}{ Frequency } & \multicolumn{3}{|c|}{ Concreteness } \\
\hline & & $\begin{array}{l}\overline{V H C} \\
(>6 \cdot 0)\end{array}$ & $\begin{array}{c}M H C \\
(4 \cdot 58-6)\end{array}$ & $\begin{array}{c}L C \\
(<4.58)\end{array}$ \\
\hline \multirow[t]{2}{*}{ PS } & AA & $94(31)$ & $63(16)$ & $33(12)$ \\
\hline & $10-50$ & $54(41)$ & $39(28)$ & $6(33)$ \\
\hline KF & $\begin{array}{l}\text { AA } \\
10-50\end{array}$ & $\begin{array}{l}90 \\
65\end{array}$ & $\begin{array}{l}67 \\
28\end{array}$ & $\begin{array}{r}43 \\
3\end{array}$ \\
\hline
\end{tabular}

other deep dyslexics; the ones read correctly averaged 3.7 letters in length, those she failed averaged 5.4 letters. $7 / 13$ numbers were read correctly. She read none of the non-words correctly and made nine substantive errors, for example maiten $\rightarrow$ made; marone $\rightarrow$ harbour.

Combining corpus I (words only) with another containing responses to 200 of the Brown and Ure words used by Coughlan ${ }^{17}$ (Corpus II) she made a total of 235 errors. The breakdown of these errors using a classification based on that of Shallice and Warrington 10 is:

$\begin{array}{lrr}\text { Visual (eg bright } \rightarrow \text { bride) } & 121 & (51 \%) \\ \begin{array}{l}\text { Derivational (eg clean } \rightarrow \text { cleaning) } \\ \text { Semantic (eg pirate } \rightarrow \text { sailor) }\end{array} & 20 & (9 \%) \\ \begin{array}{l}\text { Visual and /or Semantic } \\ \quad(\text { eg lose } \rightarrow \text { last) }\end{array} & 23 & (10 \%) \\ \begin{array}{l}\text { Visual and then Semantic } \\ \quad(\text { eg threat } \rightarrow \text { thimble) }\end{array} & 17 & (7 \%) \\ \begin{array}{l}\text { Neologisms (eg habit } \rightarrow \text { hab) } \\ \text { Other (eg cabbage } \rightarrow \text { captain, }\end{array} & 7 & (3 \%) \\ \quad \text { wicked } \rightarrow \text { think) } & 3 & (1 \%)\end{array}$

This error pattern is characteristic of deep dyslexic patients. Her rate of semantic errors is rather low when compared with that of some other deep dyslexics and the rate of visual errors is rather high (see Shallice and Warrington ${ }^{3}$ ). The visual errors occur more frequently on the abstract words. Corpus II had been selected so as to contain 100 words below the median concreteness of the Brown and Ure words and 100 above. Twenty-one of the visual errors were from the former group as opposed to only seven in the latter, significantly less $\left(\chi^{2}=6.04, p<0.02\right)$. (Only one was in the highest quartile.) The responses made in the visual errors also tended to be more imageable than the stimulus words. Twelve normal subjects rated the 116 visual error responses that occurred on Brown and Ure words (one omitted by error) together with the stimuli for imageability using a seven point scale. The responses were nearly significantly the more imageable (Wilcoxon $z=1 \cdot 59$, $\mathrm{p}<0 \cdot 06,1$ tail).

On a lexical decision task using 40 four to eight letter words and 40 equivalent pronounceable nonwords she made 16 errors (nine on words and seven on non-words). However on an auditory version she made 11 errors (six on words and five on non-words) so the difficulty was not specific to the visual modality. On a (visual) lexical decision task with function words she made $7 / 50$ errors, none being on the $13 / 25$ words she could read.

2 The impairment of reading by phonological recoding One essential prerequisite for assigning a patient's reading deficit to the category of deep dyslexia is that the ability to read by means of a phonological transformation be gravely impaired. The ability of PS to read by recoding words into a phonological form was assessed by the reading of non-words (see 1 above), by the distinguishing of rhymes from non-rhymes (following Patterson and Marcel, ${ }^{18}$ and by auditory-visual matching (following Saffran and Marin, ${ }^{19}$ and Patterson ${ }^{20}$ ).

Forty-eight pairs of four-letter words were presented, the visual similarity of each pair and whether they rhymed being varied orthogonally. An equal number were presented auditorily. PS made one error with auditory presentation on a rhyming judgment task. With visual presentation, her performance was at chance on the rhyming judgment task $(13 / 24 \mathrm{v} 12 / 24)$, being virtually entirely determined by visual similarity.

Tests of auditory-visual matching were given to PS using one target and four distractors presented visually and the target presented auditorily. With word stimuli, the distractors had either the same beginning as the target (for example pro) or the same ending (for example ant). With common endings she scored 13/15 correct; with common beginnings she scored only $5 / 15$ correct, significantly less (Fisher Exact $\mathrm{p}<0.01$ ). With four- to eight-letter pronounceable non-words and the target very dissimilar to all four distractors (for example tambote-wickle etc) she scored $10 / 10$, but if one distractor had a common beginning (for example chaggin-chaspite) she scored only 6/10; when she was wrong she always chose the visually similar distractor.

All these results indicate that her ability to use the phonological recording method of reading is indeed gravely impaired. The matching results indicate that some very minimal ability remains. It is unclear what stage of phonological recoding is impaired. However her ability to blend two- and three-syllable words from their auditorily presented individual syllables was impaired $(12 / 14$ and $9 / 14$ correct, respectively).

3 Word comprehension experiments Three types of tests of word comprehension have been performedpicture-name matching using the principle of the Peabody Test, ${ }^{21}$ synonym matching as employed by Coughlan and Warrington ${ }^{11}$ and semantic categorisation as used by Warrington and Shallice. ${ }^{22}$ In most cases the experiment was performed using both auditory and visual presentation, so as to compare the 
relative capacities for word comprehension in the two modalities.

Using strict scoring procedures for the Peabody Test she obtained a raw score of 57 on the written version of set $A$ and a raw score of 108 on the spoken version of set $B$. Considering the first 100 items (going past the basal cut-off where necessary), she scored $73 / 100$ on the written version compared with $94 / 100$ on the spoken, significantly different $\left(\chi^{2}=14.5, \mathrm{p}<0.011 \mathrm{df}\right)$. (On a French version of set $B$ presented in the spoken mode she obtained a score of 32.) On a four-choice test based on the same principles but using somewhat more difficult words (Shallice and McGill, unpublished) (for example, apprehension, outcome, sling) she scored 56/75 with auditory presentation. A written version of this test was abandoned after 35 items, as her performance of 14/35 (significantly worse than auditory (McNemar Test $\left.\chi^{2}=7 \cdot 12,1 \mathrm{df}, \mathrm{p}<0 \cdot 01\right)$ ) was little better than chance and she was finding the test distressing. On the Coughlan and Warrington 11 synonyms test in which a word has to be paired with one of two alternatives, for example "Does rely mean depend or dry" she scored $18 / 30$ with visual presentation and $25 / 30$ with auditory, marginally significantly better (McNemar Test $\left.\chi^{2}=3 \cdot 27,1 \mathrm{df}, \mathrm{p}<0 \cdot 1\right)$. With the exception of the Peabody test which contains many very easy concrete words she scored at very nearly chance level with visual presentation on these tests and fairly well with auditory presentation.

A large number of categorisation tests based on the method used by Warrington and Shallice ${ }^{22}$ were employed. In all of them the patient had to attempt to read each word (if presented visually) or if the word could not be read she had to assign it to one or other of two semantic categories. In most tests the words were also presented auditorily for cate- gorisation. Presentation of the word in the two modalities was separated in time, and either visual always preceded auditory (V) or an ABBA design was used (D). The following category discriminations were employed:

(1) Boys names versus girls names (using $150 \mathrm{~ms}$ exposure duration with tachistoscopic presentation)

(2) Surnames versus forenames

(3) Subjects (for example, latin, mathematics) versus measurements (for example, acre, ounce)

(4) Pleasant words (for example, soothe, agreement) versus unpleasant words (for cxample, angry, meanness)

(5) Money-related words (for example, expense, earnings) versus thought-related words (for example idea, knowledge).

The results are shown in table 2 . On some tests performance with auditory presentation is very good, but with visual presentation it is very poor; for words that cannot be read aloud categorisation performance is not significantly better than chance. On other tests with very comparable auditory performance, visual performance is nearly as good.

\section{Discussion}

The pattern of reading disability shown by PS conforms to the generally accepted criteria for deep dyslexia. ${ }^{2}{ }^{3}$ Her ability to read using a phonological route (that is "sounding out") was grossly impaired, as shown by her inability to read pronounceable nonsense words, her inability to say whether visually presented words rhyme and her inability to determine more than the initial sound

Table 2 Two-choice categorisation performance of PS on different types of categorical judgment. $I$ and II refer to testing periods, separated by an interval of one year. For tests labelled $V$ visual presentation preceded auditory, and for those labelled D an ABBA design was used for the different modalities. The "chance" column gives the mean and standard deviation of the distribution of correct responses for unread words to be expected if categorisation performance of such words is at chance. The "read" column includes derivational errors, semantic errors and paraphasic responses where appropriate

\begin{tabular}{|c|c|c|c|c|c|c|}
\hline & & \multicolumn{4}{|c|}{ VISUAL } & \multirow{2}{*}{$\frac{\text { AUDITORY }}{\text { Categorisation performance }}$} \\
\hline & & \multirow{2}{*}{$\begin{array}{l}\text { No of } \\
\text { words } \\
\text { read }\end{array}$} & \multicolumn{3}{|c|}{ Categorisation performance } & \\
\hline & & & $\begin{array}{l}\text { Unread } \\
\text { words } \\
\text { only }\end{array}$ & (Chance) & $\begin{array}{l}\text { All } \\
\text { words }\end{array}$ & $\begin{array}{l}\text { All } \\
\text { words }\end{array}$ \\
\hline \multirow[t]{2}{*}{ Pleasant/unpleasant } & IV & - & - & & $30 / 60$ & $58 / 60$ \\
\hline & IID & $14 / 60$ & $28 / 46$ & $(23 \pm 3 \cdot 4)$ & $42 / 60$ & $57 / 60$ \\
\hline Financial/thought & IIV & $4 / 40$ & $17 / 36$ & $(18 \pm 3 \cdot 0)$ & $21 / 40$ & $40 / 40$ \\
\hline \multirow[t]{2}{*}{ Surnames/forenames } & I & $18 / 40$ & $21 / 22$ & $(11 \pm 2 \cdot 3)$ & $38 / 40$ & - \\
\hline & IID & $15 / 30$ & $12 / 15$ & $(7 \cdot 5 \pm 1.9)$ & $27 / 30$ & $29 / 30$ \\
\hline \multirow{4}{*}{ Subjects/measurements } & I & $25 / 45$ & $16 / 20$ & $(10 \pm 2 \cdot 2)$ & $41 / 45$ & - \\
\hline & I & $8 / 30$ & $18 / 22$ & $(11 \pm 2 \cdot 3)$ & $26 / 30$ & - \\
\hline & & $15 / 50$ & $26 / 35$ & $(17 \cdot 5 \pm 3 \cdot 0)$ & $41 / 50$ & - \\
\hline & IID & $15 / 50$ & $28 / 35$ & $(17 \cdot 5 \pm 3 \cdot 0)$ & $42 / 50$ & $47 / 50$ \\
\hline
\end{tabular}


ing auditory-to-visual matching. She also displayed the other characteristic features of deep dyslexia, namely the production of semantic, derivational and visual errors and sensitivity to both concreteness (concrete words read better than abstract ones) and part of speech (concrete nouns read better than adjectives which are read better than verbs). The one possible difference from previously reported deep dyslexic reading was her relatively good performance on function words, but she had received extensive training on these words.

The major aim of the research with PS was to investigate word comprehension. However information relevant to two proposed explanations of the syndrome is available. Firstly, it would seem that Richardson's imagery theory, ${ }^{23}$ in which it is presupposed that deep dyslexics read through the word triggering a visual image, is not supported by our results. Presumably the theory would predict that forenames and surnames would produce similar visual images that would not be differentiable without the aid of auditory information. The fact that PS can distinguish competently between surnames and forenames which she cannot read aloud therefore argues against Richardson's hypothesis. Secondly our data on the very strong left-ear effect in PS's performance on dichotic listening could, if Kimura's theory ${ }^{24}$ of dichotic listening is accepted, be taken as providing indirect support for the theory that deep dyslexia represents right-hemisphere reading. An alternative account of this finding in terms of attentional factors following the work of Morais and Bertelson ${ }^{25}$ on normal subjects would, however, also be plausible, especially in view of PS's short-term memory impairment; the dichotic listening finding would then correspond to a material-specific right-sided neglect.

In the investigation of PS's word-comprehension skills matching and categorisation tasks were employed, in both auditory and visual modalities. On most of these tests her performance given auditory presentation was nearly perfect and with visual performance near chance unless she could read the word. On three tests, where she was required to sort words into two categories which clearly exist as such in languagesurname/forename, boys/girls name and subject/ measurement-she performed nearly as well visually as auditorily. However, on two formally similar tasks, where the discrimination did not fit so clearly with the structure of the natural categories existing in language-pleasant/unpleasant words and thought/financial words, she performed at chance on visual presentation. The words use in the different categorisation tasks could not be drawn from a common pool so semantic differences between the classes of words used might be a relevant factor. The different pattern of performance between different tests seems, however, unlikely to be a chance phenomenon; the tests were repeated after a year's gap and very similar results obtained.

At first sight it may seem that such a pattern of results does not unambiguously favour either the semantic attainment hypothesis, which predicts a relative deficit on visual input, or the naming hypothesis, which predicts no such deficit. However on one version of the "semantic attainment" hypothesis good performance on certain types of visual word categorisation tasks would be expected. This would be the case if the deficit for certain classes of words was of the type found in a patient studied by Warrington and Shallice ${ }^{22}$ in which broad categorical information was available about written words but the precise meaning could not be attained, a difficulty described as "semantic access dyslexia". Indeed the categorisation of words she could not read into well defined categories was the only type of task at which she performed as well with visual presentation as with auditory.

By contrast, if the deficit were of the naming type a reduced level of visual categorisation performance with respect to auditory performance can only be explained through assuming an additional deficit to some stage in the processing of the written word which is specific to visual input. If this is not to involve the semantic attainment process, the deficit would have to be at some pre-semantic stage, say, at the level of the analysis of the visual word-form (see Warrington and Shallice ${ }^{26}$ ). Yet such an associated deficit would not seem to be able to account for different levels of performance between different categorisation tasks using written words; in particular the good performance on tachistoscopic categorisation of boys/girls names. Also if a sufficiently severe additional pre-semantic deficit be assumed such that comprehension performance on abstract words can be at chance, one cannot explain how concrete words can be read.

It therefore appears that the semantic attainment hypothesis is the most plausible and that PS cannot attain the precise semantic representation of more abstract words given visual input, even though she can given auditory input. In conjunction with an inability to use the phonological route method of reading, is a deficit in 
attaining the precise semantic representation of more abstract words from visual input sufficient to account for the symptom pattern of deep dyslexia? The findings of Morton and Patterson ${ }^{27}$ on the inability of some deep dyslexics to process the syntactic features of words cannot be explained in this way and would require at least a parallel deficit with respect to syntax. However all the other characteristics of the syndrome are compatible with such an explanation, although for the effect of part-of-speech and the existence of semantic and derivational errors, there is no evidence that a semantic attainment explanation is preferable to the "naming" one. Visual errors can be explained either as part of the semantic attainment theory 1019 or as in the "naming" theory through an additional deficit. ${ }^{9}$ However the semantic attainment theory, but not the naming one, predicts that visual errors occur on the more abstract words, which is what happens in PS and has been observed in other patients. ${ }^{3} 728$

Would the semantic attainment theory explain the symptoms exhibited by all deep dyslexics? This seems unlikely as major differences in the comprehension abilities of deep dyslexics do appear to exist. The performance of other deep dyslexics on categorisation tests similar to the ones used here has not been reported. However both VS (Saffran and Marin, ${ }^{19}$ and PW (Patterson, $\left.{ }^{9}\right)$ performed well on the Peabody picturevocabulary test, visually as well as auditorily, a different pattern from PS. Moreover these two patients both perform very well on lexical decision $^{9} 1819$ and as this task appears to involve semantic processing ${ }^{29}$ this provides further evidence that their defect is not one of semantic attainment. The possibility must therefore remain, as suggested by Shallice and Warrington, ${ }^{3}$ that deep dyslexic patients are not functionally homogeneous. However it would appear that, at the very least, a subclass of deep dyslexics exists whose deficits can be explained in terms of a dual deficit of phonological recoding and of the attainment of the semantic representation of certain semantic classes of word from visual input only.

We thank Dr David Jenkins, Medical Director of the Wolfson Rehabilitation Centre for permission to investigate this patient and to report our findings and $\mathrm{Mr} \mathrm{HH}$ Gossman of Plymouth General Hospital for permission to repont the results of his neurosurgical investigations. This research was partially supported by a grant from the Medical Research Council to Dr E K War- rington at The National Hospital. We thank Karalyn Patterson and Elizabeth Warrington for their comments on an earlier draft of this paper.

\section{References}

1 Marshall JC, Newcombe F. Syntactic and semantic errors in paralexia. Neuropsychologia 1966; 4:169-76.

2 Coltheart M. Deep dyslexia: a review of the syndrome. In: Coltheart $\mathrm{M}$, Patterson $\mathrm{KE}$, Marshall J, eds. Deep Dyslexia. London: Routledge, 1980 .

3 Shallice T, Warrington EK. Single and multiple component central dyslexic syndromes. In: Coltheart M, Patterson KE, Marshall J, eds. Deep Dyslexia. London: Routledge, 1980.

4 Allport DA. On knowing the meaning of words we are unable to report: the effects of visual masking. In: Dornic S, ed. Attention and Performance VI. London: Academic Press, 1977.

5 Saffran EM, Schwartz MF, Marin OSM. Semantic mechanisms in paralexia. Brain Lang 1976; 3: 255-65.

6 Marcel AJ, Patterson KE. Word recognition and production: reciprocity in clinical and normal studies. In: Requin J, ed. Attention and Performance VII. Hillsdale: Erlbaum, 1978.

7 Coltheart M. Deep dyslexia: a right hemispherc hypothesis. In: Coltheart M, Patterson KE, Marshall J, eds. Deep Dyslexia. London: Rou:ledge, 1980.

8 Beauvois M-F, Dérouesné J. Phonological alexia: three dissociations. J Neurol Neurosurg Psychiatry 1979; 42:1115-24.

9 Patterson KE. What is right with deep dyslexic patients? Brain Lang 1979; 8:111-29.

10 Shallice T, Warrington EK. Word recognition in a phonemic dyslexic patient. $Q J$ Exp Psychol 1975; 27:187-99.

11 Coughlan AK, Warrington EK. Word comprehension and word retrieval in patients with localised cerebral lesions. Brain 1978; 101:163-85.

12 Lesser $\mathbf{R}$. Verbal comprehension in aphasics: an English version of three Italian tests. Cortex 1974; 10:247-63.

13 De Renzi E, Vignolo LA. The Token Test: a sensitive test to detect receptive disturbances in aphasics. Brain 1962; 85:665-78.

14 Warrington EK, Sanders HI. The effect of prior learning on subsequent retention in amnesic patients. Neuropsychologia 1974; 12:419-28.

15 Warrington EK, Taylor A. The contribution of the right parietal lobe to object recognition. Cortex 1973; 9:152-64.

16 Brown WP, Ure DMJ. Five rated characteristics of 650 word association stimuli. Br J Psychol 1969; 60:223-50.

17 Coughlan AK. An investigation of selected verbal skills and verbal memory following left- 
hemisphere lesions. PhD Thesis, University of London, 1976.

18 Patterson KE, Marcel AJ. Aphasia, dyslexia and the phonological coding of written words. $Q J$ Exp Psychol 1977; 29:307-18.

19 Saffran EM, Marin OSM. Reading without phonology: evidence from aphasia. $Q J$ Exp Psychol 1977; 29:515-25.

20 Patterson KE. Phonemic dyslexia: errors of meaning and meaning of errors. $Q J$ Exp Psychol 1978; 30:587-601.

21 Dunn LM. Peabody Picture Vocabulary Test. Circle Pines: American Guidance Service, 1959.

22 Warrington EK, Shallice T. Semantic access dyslexia. Brain 1979; 102:43-63.

23 Richardson JTE. The effect of word imageability in acquired dyslexia. Neuropsychologia 1975; 13: 281-8.

24 Kimura D. Somé effects of temporal-lobe damage on auditory perception. Can J Psychol 1961; 15: 156-65.

25 Morais J, Bertelson P. Spatial position versus ear of entry as determinant of auditory laterality effects: a stereophonic test. J Exp Psychol (Hum Percept) 1975; 1:253-62.

26 Warrington EK, Shallice T. Word-form dyslexia. Brain 1980; 103:99-112.

27 Morton J, Patterson KE. "Little words-no!" In: Coltheart M, Patterson KE, Marshall J, eds. Deep Dyslexia. London: Routledge, 1980.

28 Morton J, Patterson KE. A new attempt at an interpretation or an attempt at a new interpretation. In: Coltheart M, Patterson KE, Marshall J, eds. Deep Dyslexia. London: Routledge, 1980.

29 James CT. The role of semantic information in lexical decisions. J Exp Psychol (Hum Percept) 1975; 1:130-6. 